\title{
H. pylori infection and gastric cancer in Bangladesh: a case-control study
}

Khandker Kawser Sarker, FCPS, MS ${ }^{a}$, Md. Jahangir Kabir, FCPS,MS, MRCS ${ }^{a}$,

A.K.M. Minhaj uddin Bhuyian, FCPS, MS, MRCS ${ }^{a}$, Md. Shahjadul Alam, FCPS, MS ${ }^{a}$,

Fazle Rabbi Chowdhury, FCPS, MSC ${ }^{b}$, M. Abdul Ahad, FCPS, MD' ${ }^{c}$ Md. Anisur Rahman, FCPS ${ }^{d}$,

M. Mizanur Rahman, FCPS ${ }^{a, \star}$

Background: Like that of other Asian countries gastric cancer (GC) is also a leading cancer in Bangladesh and also a cause for cancer-related mortality. Infection with Helicobacter pylori (H. pylori) is the strongest recognized risk factor for gastric adenocarcinoma. The infection is also prevalent in common people. This case-control study was carried out to find an association between $\mathrm{GC}$ and $H$. pylori infection in the community.

Materials and Methods: To evaluate association of $H$. pylori and carcinoma of stomach this study was conducted at National Institute of Cancer Research \& Hospital, Dhaka from January 2013 to December 2014. H. pylori status was determined serologically by using $\mathrm{H}$. pylori kit in the department of Biochemistry laboratory of Bangabandhu Sheikh Mujib Medical University. In total, 114 patients with GC and 520 patients not having GC were studied as controls. Logistic regression method was used to calculate the odds ratio.

Results: Significantly more patients in the case group (86.8\%) were found to be seropositive for $\mathrm{H}$. pylori antigen in contrast to the control group (67.5\%). All of the cases in the present study were in advanced stage. No significant association between H. pylori seropositivity and tumor location was found. It was noted that undifferentiated gastric carcinoma had slightly more association with $H$. pylori infection. Younger $H$. pylori-infected patients had been found to be at higher relative risk for $\mathrm{GC}$ than older patients.

Conclusion: As there is a strong association found between GC and $H$. pylori infection special emphasis to eradicate $H$. pylori infection might reduce the incidence of this dreadly disease.

Keywords: Gastric cancer, H. pylori infection, Case-control study

Gastric cancer (GC) is one of the important malignancies that causes widespread morbidity and mortality. Like that of other Asian countries GC is also a leading cancer in Bangladesh, in male it ranks after lung cancer. So far, infection with Helicobacter pylori (H. pylori) is the strongest recognized risk factor for $\mathrm{GC}^{[1]}$. Chronic infection with the bacterium H. pylori causes noncardia gastric carcinoma and low-grade B-cell MALT lymphoma ${ }^{[2]}$. It was estimated that worldwide 660,000 cases of cancer in the year

${ }^{a}$ Department of Surgical Oncology, National Institute of Cancer Research and Hospital, Mohakhali, Dhaka, 'DMAG Osmani Medical College, Sylhet, cKhulna Medical College and ${ }^{d}$ Popular Medical College Hospital, Dhaka, Bangladesh

This manuscript has been peer reviewed.

Sponsorships or competing interests that may be relevant to content are disclosed at the end of this article.

${ }^{*}$ Corresponding author. Address: Department of Surgical Oncology, National Institute of Cancer Research and Hospital, Mohakhali, Dhaka, 1012 Bangladesh. Tel: + (88) 01711161199. E-mail address: mizannicrh@gmail.com (M.M. Rahman).

Copyright $\odot 2017$ The Authors. Published by Wolters Kluwer Health, Inc. on behalf of IJS Publishing Group Ltd. All rights reserved. This is an open-access article

distributed under the terms of the Creative Commons Attribution-Non CommercialNo Derivatives License 4.0 (CCBY-NC-ND), where it is permissible to download and share the work provided it is properly cited. The work cannot be changed in any way or used commercially without permission from the journal.

International Journal of Surgery Oncology (2017) 2:e44

Received 7 August 2017; Accepted 15 August 2017

Published online 16 October 2017

http://dx.doi.org/10.1097//I9.00000000000000044
2008 were attributable to $H$. pylori, corresponding to $32.4 \%$ of the 2 million cancer cases attributable to infectious agents and $5.2 \%$ of the 12.7 million total cancer cases that occurred worldwide $^{[3]}$. The vast majority of the cancers attributable to H. pylori $(650,000)$ were noncardia gastric carcinoma ${ }^{[4]}$.

The severity and long-term outcome of this infection is modulated by an increasing list of bacterial, host, and environmental factors, which interplay in a complex manner. Identification of individuals at high risk for GC that may enter a surveillance program and intervention during the precancerous process is the most suitable strategy for decreasing mortality due to this malignancy. The 21st century has brought more attention to infectious agents and chronic active inflammation as primary causes of some cancers. Although the absolute numbers of GC are decreasing in western countries, some studies suggest different patterns in various age groups, with a particular significant increase in premalignant lesions and GC among younger patients ${ }^{[5]}$.

It is also reported that colonization with $H$. pylori is not the sole determinant for the development of GC. Risk modulators are in particular related to lifestyle. The recognition of these risk modulators determines the options for prevention and intervention to decrease the incidence of $\mathrm{GC}^{[6]}$. In Bangladesh some studies are carried out regarding association of $H$. pylori with dyspepsia $^{[7]}$. But there is no clear sorological evidence found linking to GC. In this study we examined the serological association with GC patients as a case control manner. 


\section{Materials and methods}

This case-control study was carried out at the department of Surgical Oncology of National Institute of cancer research and hospital from January 2013 to December 2014. Purposive sampling technique was applied to select cases for this study. All patients with endoscopically and histopathologically proven adenocarcinoma of stomach admitted under surgical Oncology department of NICRH were included in the study. Controls were selected from patients having no cancer on endoscopy, matched to cases by age, sex, socioeconomic status. All cases and controls were evaluated by history, examinations, and investigations. GC cases were classified as cardia or proximal cancer if their location was in gastrooesophageal junction and fundus and as noncardia cancer otherwise, distal cancer if the tumor location was below the mid body or in the antrum. GC was also divided into early and advanced cancer, after pathologic examination following endoscopic or surgical resection. Early gastric cancer (EGC) is defined as a tumor that was confined to the mucosa or submucosa regardless of lymph node involvement, and advanced gastric cancer (AGC) was defined as a tumor that invaded beyond the submucosa. In patients who did not undergo resection due to metastatic GC, tumor stages were determined by endoscopic and computer tomographic findings. GCs were classified histopathologically according to Lauren's system (intestinal, diffuse, and mixed type) and on the grading (well differentiated, moderately differentiated, undifferentiated). Peroperative analysis of most of the cases were performed to see tumor location invasion to adjacent organ, lymph node involvement, ascites, hepatic metastasis, and peritoneal seedlings. Postoperative specimen was sent for histologic examination to see tumor location, stage, grade, and Lauren's type. A blood sample $(5 \mathrm{~mL}$ without preservative) was obtained from each participant, anti-H. pylori IgG was measured using H. pylori kit at Bangabandhu Sheikh Mujib Medical University Laboratory. All participants were categorized as seropositive and seronegative for $H$. pylori according to selective cutoff value of IgG. Categorical data were expressed as number and percentage and were compared using the $\chi^{2}$ test. Multivariate logistic regression was done to analyze the risk for $H$. pylori infection; $P$-value of $\leq 0.05$ was considered as significant. For control, adult people above 30 years of age, who attended endoscopical examination for nonspecific abdominal pain were included in this series. Subjects were selected from 3 centers across the country, from Dhaka, Khulna, and Sylhet. None had any feature of malignancy or any pathology by endoscopy.

\section{Results}

In this study, 114 GC patients and 520 control subjects were studied. The mean age of the control patients was $46.01( \pm 13.56)$ years and that of the case patients was $51.11( \pm 12.65)$ years. By category, 81 patients and 309 control patients were males. Additional salt intake and smoking habits were significantly more prevalent in the case group $(P<0.05)$. Case patients were significantly less educated than control patients $(P<0.05)$ (Table 1$)$. In the case group 99 patients $(86.8 \%)$ exhibited seropositivity against $H$. pylori infection, whereas in the control group this percentage was 67.5 . This difference of seropositivity was statistically significant (Table 2 ). Table 3 shows the cross-tabulation of the case patients by the $H$. pylori seropositivity and morphology. No significant association was noted between tumor
Table 1

Baseline characteristics of the patients.

\begin{tabular}{lrrr}
\hline & \multicolumn{3}{c}{ Category } \\
\cline { 2 - 3 } Baseline & $\begin{array}{c}\text { Control (N=520 With } \\
\text { Characteristics }\end{array}$ & $\begin{array}{c}\text { Case (N=114 With } \\
\text { Percentage) }\end{array}$ & $\boldsymbol{P}$ \\
\hline Age (mean \pm SD) $(y)$ & $46.01(13.56)$ & $51.11(12.65)$ & 0.654 \\
Male sex & $309(59.4)$ & $81(71.1)$ & 0.082 \\
Additional salt intake & $113(21.7)$ & $44(38.6)$ & 0.042 \\
Ever smoking & $140(26.9)$ & $60(52.6)$ & 0.031 \\
Family income/mo & $201(78.7)$ & $87(76.3)$ & 0.254 \\
$\quad(>10,000$ taka) & & & \\
SSC or above level & $268(51.5)$ & & \\
$\quad$ education & & & \\
\end{tabular}

${ }^{*} 10,000$ taka $=120$ USD.

SSC indicates secondary school certificate.

location, Lauren tumor type, tumor grading, and H. pylori seropositivity. Cross-tabulation of the case patients by location, grade, and the type of the carcinoma is presented in Table 4. It was found that intestinal type is mostly seen in distal gastric carcinoma while most of the intestinal carcinoma was well to moderately differentiated. Diffuse or mixed type tumors were mainly poorly differentiated. These differences were statistically significant $(P<0.05)$. Multivariate logistic regression analysis of risk for $H$. pylori infection was performed and the result presented in Table 5. The odds ratio (OR) of ever smokers was 2.253 higher than nonsmokers. Age below 50 years, male, and additional salt intake were other significant risk factors. Table 6 depicts the ORs and $95 \%$ confidence intervals for the association between $H$. pylori infection and GC by subgroup. It was found that ulceroproliferative type and poorly differentiated carcinoma had higher ORs in contrast to tumor location and type.

\section{Discussion}

Bangladesh is a South Asian developing country, where the rate of $H$. pylori infection is particularly high. Mahalanabis et $\mathrm{al}^{[8]}$ in a study of $13 \mathrm{C}$-urea breath test also reported that the prevalence of H. pylori was $63 \%$ in infants aged $1-3$ months, $33 \%$ in $10-$ 15 -month-old children, and $84 \%$ in 6-9 years old. Moreover, the overall $H$. pylori prevalence in other Asian countries including, India $(79 \%$ by ELISA), Pakistan $[84 \%$ by polymerase chain reaction (PCR)], and Japan (41\% by measuring urinary levels of anti-H. pylori antibody) was also reported to be high ${ }^{[9]}$. In Europe $(40 \%)$ and the United States (40\%), a significantly lower prevalence rate of $H$. pylori was observed ${ }^{[10]}$. High $H$. pylori infection rates in developing countries compared with the developed world may be the consequence of poor socioeconomic conditions and unhygienic life styles ${ }^{[9]}$.

Table 2

Distribution of $\boldsymbol{H}$. pylori seropositivity among the respondents.

\begin{tabular}{|c|c|c|c|c|}
\hline \multirow[b]{2}{*}{ H. pylori Seropositivity } & \multicolumn{2}{|c|}{ Category } & \multirow[b]{2}{*}{$x^{2}$} & \multirow[b]{2}{*}{$P$} \\
\hline & Control $(\mathrm{N}=520)$ & Case $(\mathrm{N}=114)$ & & \\
\hline Present & $351(67.5)$ & $99(86.8)$ & 16.98 & $<0.001$ \\
\hline Absent & 169 (32.5) & $15(13.2)$ & & \\
\hline Total & $520(100.0)$ & $114(100.0)$ & & \\
\hline
\end{tabular}

Percentages are given in the parenthesis. 
Table 3

Cross-tabulation of the case patients by the of $\boldsymbol{H}$. pylori seropositivity and morphology $(\mathrm{N}=114)$.

\begin{tabular}{lrrr}
\hline & \multicolumn{2}{c}{ H. pylori Seropositivity } & \\
\cline { 2 - 3 } Morphology & Present & Absent & P \\
\hline Tumor location & & & \\
$\quad$ Proximal & $28(28.3)$ & $3(20.0)$ & 0.502 \\
$\quad$ Distal & $71(71.7)$ & $12(80.0)$ & \\
$\begin{array}{l}\text { Type } \\
\text { Intestinal }\end{array}$ & $74(74.7)$ & $10(66.7)$ & 0.507 \\
$\quad$ Diffuse/mixed & $25(25.3)$ & $5(33.3)$ & \\
Grading & & & \\
$\quad$ Well to moderately differentiated & $69(69.7)$ & $11(73.3)$ & 0.774 \\
$\quad$ Poorly differentiated & $30(30.3)$ & $4(26.7)$ & \\
\hline
\end{tabular}

Percentages are given in the parenthesis.

We found that among 111 patients, $60(54.05 \%)$ were positive by the CLO test and $54(48.65 \%)$ were positive by PCR. Although PCR is more sensitive and more specific than the CLO test, this study produced unsatisfactory PCR results compared with the results of CLO test ${ }^{[11]}$.

Significantly more patients in the case group $(86.8 \%)$ were seropositive for $H$. pylori antigen in contrast to the control group $(67.5 \%)$. This result supports the conclusion by the IARC that infection with $H$. pylori is a risk factor for GC in humans. All of the cases in the present study were in advanced stage. However, in previous analyses, the prevalence of $H$. pylori infection was significantly higher in patients with EGC than in those with $\mathrm{AGC}^{[12,13]}$. Most untreated EGCs are reported to progress to AGC within 4-5 years ${ }^{[14]}$. The lower frequency of $H$. pylori $\mathrm{IgG}$ antibodies in AGC may result from a decrease in antibody titer, due to the development of advanced $H$. pylori-associated atrophic gastritis concomitant with age.

We found no significant association between $H$. pylori seropositivity and tumor location though a strong positive association has been reported between $H$. pylori seropositivity in gastric noncardia adenocarcinoma ${ }^{[13,15,16]}$. Studies have found a null $^{[13,16]}$ or inversely associated relationship ${ }^{[15]}$ between anti- $H$. pylori seropositivity and gastric cardia cancer. This association shows substantial geographic variation. Most studies in Asian populations have found a positive association between $H$. pylori seropositivity and cardia cancer ${ }^{[12,17]}$, whereas most studies in western populations have found no association or an inverse association $^{[15,18,19]}$. This discrepancy may have been due to the classification in western studies of some patients with esophageal adenocarcinoma as having gastric cardia cancer ${ }^{[7,15]}$.

\section{Table 4}

Cross-tabulation of the case patients by location, grade, and the type of the carcinoma.

\begin{tabular}{lrrrrr} 
& \multicolumn{2}{c}{ Tumor Types [n (\%)] } & & \\
\cline { 2 - 3 } Morphology & Intestinal & Diffuse/Mixed & $\mathbf{X}^{\mathbf{2}}$ & $\boldsymbol{P}$ \\
\hline $\begin{array}{l}\text { Tumor location } \\
\quad \text { Proximal }\end{array}$ & $17(54.8)$ & $14(45.2)$ & 6.159 & 0.013 \\
$\quad$ Distal & $65(78.3)$ & $18(21.7)$ & & \\
$\begin{array}{l}\text { Grading } \\
\quad \text { Well to moderately differentiated }\end{array}$ & $71(87.5)$ & $9(12.5)$ & 31.398 & $<0.001$ \\
$\quad$ Poorly differentiated & $13(38.2)$ & $21(61.8)$ & & \\
\hline
\end{tabular}

Table 5

Multivariate logistic regression analysis of risk for $\boldsymbol{H}$. pylori Infection.

\begin{tabular}{lccc}
\hline & & \multicolumn{2}{c}{$\mathbf{9 5 \%}$ CI } \\
\cline { 3 - 4 } Risk for $\boldsymbol{H}$. pylori Infection & OR & Lower & Upper \\
\hline Age $(<50$ y) & 1.385 & 0.624 & 2.501 \\
Sex (male) & 1.401 & 0.433 & 4.254 \\
Additional salt intake & 1.421 & 0.771 & 2.523 \\
Ever smoker & 2.312 & 1.058 & 4.812 \\
Familial income $(<10,000)$ & 1.151 & 0.511 & 2.492 \\
Education (SSC or above) & 0.785 & 0.452 & 1.617 \\
\hline
\end{tabular}

$\mathrm{Cl}$ indicates confidence interval; OR, odds ratio; SSC, secondary school certificate.

H. pylori has been reported to be a causal factor in the atrophic gastritis-intestinal metaplasia-intestinal type of GC sequence, hypothesized by Correa ${ }^{[20]}$. The prevalence of $H$. pylori infection seems to be greater in intestinal type than in diffuse type $\mathrm{GCs}^{[21,22]}$. However, most comprehensive studies have shown that there is no difference in $H$. pylori seroprevalence between these 2 types ${ }^{[13,15,16]}$, a finding consistent with the present study. GC can be classified as differentiated or undifferentiated carcinoma according to Japanese classification ${ }^{[23]}$. We found that undifferentiated gastric carcinoma had slightly more association with $H$. pylori infection. Although previous studies showed that H. pylori infection may be associated with the differentiated, but not the undifferentiated type of $\mathrm{GC}^{[24,25]}$. Moreover, a study in Japan indicated that the ORs were similar (5.8 for differentiated and 5.1 for undifferentiated type ${ }^{[26]}$ ). Younger $H$. pylori-infected patients have been found to be at higher relative risk for GC than older patients, a finding consistent with the present study. This can be explained by the lower infection rate in the younger controls, whereas the age-related prevalence of $H$. pylori infection increased significantly with the cohort effect in controls but not in cases $^{[13]}$. In addition, H. pylori prevalence was higher in younger than in older GC patients, which may be due to the spontaneous disappearance of infection caused by increased mucosal atrophy and intestinal metaplasia with advanced age, inhospitable place for $H$. pylori colonization ${ }^{[27-29]}$. Generally, patients with GC have more severe mucosal atrophy and intestinal metaplasia in the stomach than normal subjects ${ }^{[30]}$. Another hypothesis is that humoral immune response tends to decrease with age ${ }^{[31]}$, resulting in the underdetection of serum antibodies against H. pylori. Meanwhile, earlier reports showed that the prognosis of patients with early onset of GC was poor, with a short survival potential,

\section{Table 6}

Odds ratios (ORs) and $95 \%$ confidence intervals (Cls) for the association between $\boldsymbol{H}$. pylori infection and gastric cancer by subgroup.

\begin{tabular}{lccr}
\hline & & \multicolumn{2}{c}{$\mathbf{9 5 \%} \mathbf{~ C l}$} \\
\cline { 3 - 4 } Factors & OR & Lower & Upper \\
\hline Ulceroproliferative type & 2.121 & 0.314 & 11.012 \\
Location (distal) & 0.451 & 0.069 & 2.531 \\
Lauren's type (diffuse/mixed) & 0.445 & 0.051 & 3.870 \\
Grade (poorly differentiated) & 1.481 & 0.177 & 9.521 \\
\hline
\end{tabular}


especially in patients who presented with advanced gastric carcinoma $^{[32,33]}$. In a few reports, however, the prognosis of patients with early-onset GC who underwent gastrectomy was better than that of older patients ${ }^{[34]}$. Recent reports have showed no difference in surgical outcomes between older and younger patients with $\mathrm{GC}^{[35]}$. Therefore, age does not seem to be an independent risk factor for GC. Regarding to sex, hormonal difference might be an important factor for prognosis in patients with GC. Several studies found that female sex hormones and their analogs appear to be associated with gastric carcinogenesis and progression, and that pregnancy and delivery may accelerate growth of stomach cancer cells ${ }^{[36,37]}$. Further studies are needed to evaluate different outcomes between both sexes in young GC patients. A long time study performed in Finland reported that of all GCs that occurred during the 15-year follow-up among elderly men, only $11 \%$ appeared in men with healthy stomachs. The risk of stomach cancer is approximately 6 times higher among men with $H$. pyloric infection than among men with healthy stomach mucosa, and the H. pylori infection raises the GC risk similarly in the gastric cardia and in other sites of the stomach ${ }^{[38]}$.

Like that of international studies, in Bangladesh H. pylori is also found in $>80 \%$ of GC cases, again it is prevalent in the community also. To date, existing findings indicate that GC is the biological translation of carrying an infectious disease, which is interestingly preventive with anti-H. pylori regimen ${ }^{[39]}$. Therefore, as an inevitable consequence, identification of $H$. pylori colonized in people with high risk of GC is the main direction of the future research. It is postulated that if $H$. pylori can be removed from the population, it has been estimated that $\sim 75 \%$ of GC would be eliminated $^{[40]}$. So it is high time to take an initiative for anti $H$. pylori measures that will help to reduce the number of new cases.

\section{Ethical approval}

Taken from the ethical review committee of the National Institute of Cancer Research and Hospital, Dhaka.

\section{Sources of funding}

Supported by the Ministry of Science and Technology, Government of Bangladesh.

\section{Author contribution}

K.K.S.: protocol making, study designing, making data collection sheet, taking samples from patients, data compilation, and writing. M.J.K.: selection of the patients, active in surgical intervention, taking samples from the patient, collection of the samples, and data compilation. A.K.M.M.u.B.: selection of the patients, active in surgical intervention, taking samples from the patient, collection of the samples, and data collection. M.S.A.: selection of the patients, active in surgical intervention, taking samples from the patient, collection of the samples. F.R.C.: coordinating the samples for control across the different parts of the country, final data analyzing. M.A.A. and M.A.R.: helping for giving the concepts, endoscopist for patients and controls, and sample collection. M.M.R.: giving the theme, study designing, monitoring all steps like sample collection from patients and control, data analyzing, giving the final shape of the manuscript, responsible for fund collection, and corresponding author. Every author has contributed towards study designing, case selection, data collection, compiling, analysis, and writing.

\section{Conflict of interest disclosure}

The authors declare that they have no financial conflict of interest with regard to the content of this report.

\section{Research registration unique identifying number (UIN)}

Not applicable.

\section{Guarantor}

M. Mizanur Rahman.

\section{Acknowledgments}

The authors also are thankful to the doctors of Surgical Oncology department and staff who helped us with their full hands and cooperation in National Institute of Cancer Research and Hospital, Mohakhali, Dhaka. The authors are thankful to the staff of the Biochemistry department of Bangabandhu Sk Mujib Medical University for their full assistance.

\section{References}

[1] Correa P, Piazuelo MB. Natural history of Helicobacter pylori infection. Dig Liver Dis 2008;40:490-6.

[2] IARC. Monographs on the Evaluation of Carcinogenic Risks to Humans Volume 100b: A Review of Human Carcinogens: Biological Agents. Lyon: International Agency for Research on Cancer; 2012.

[3] de Martel C, Ferlay J, Franceschi S, et al. Global burden of cancers attributable to infections in 2008: a review and synthetic analysis. Lancet Oncol 2012;13:607-15.

[4] Plummer M, Franceschi S, Vignat J, et al. Global burden of gastric cancer attributable to Helicobacter pylori. Int J Cancer 2015;136:487-90.

[5] Song H, Held M, Sandin S, et al. Increase in the prevalence of atrophic gastritis among adults age 35 to 44 years old in Northern Sweden between 1990 and 2009. Clin Gastroenterol Hepatol 2015;13:1592600.

[6] den Hoed CM, Kuipers EJ. Gastric cancer: how can we reduce the incidence of this disease? Curr Gastroenterol Rep 2016;18:34.

[7] Miah MA, Rahman M, Rumi KA, et al. Prevalence of Helicobacter pylori in asymptomatic population-a pilot serological study in Bangladesh. J Epidemiol 1997;7:251-4.

[8] Mahalanabis D, Rahman MM, Sarker SA, et al. Helicobacter pylori infection in the young in Bangladesh: prevalence, socioeconomic and nutritional aspects. Int J Epidemiol 1996;25:894-8.

[9] Graham DY, Adam E, Reddy GT, et al. Seroepidemiology of Helicobacter pylori infection in India. Comparison of developing and developed countries. Dig Dis Sci 1991;36:1084-8.

[10] Shiota S, Murakami K, Takayama A, et al. Evaluation of Helicobacter pylori status and endoscopic findings among new outpatients with dyspepsia in Japan. J Gastroenterol 2009;44:930-4.

[11] Habib AM, Alam MJ, Rudra B, et al. Analysis of Helicobacter pylori prevalence in Chittagong, Bangladesh, based on PCR and CLO test. Microbiology Insights 2016;9:47-50.

[12] Kato M, Asaka M, Shimizu Y, et al. Relationship between Helicobacter pylori infection and the prevalence, site and histological type of gastric cancer. Aliment Pharmacol Ther 2004;20:85-9.

[13] Huang JQ, Sridhar S, Chen Y, et al. Meta-analysis of the relationship between Helicobacter pylori seropositivity and gastric cancer. Gastroenterology 1998;114:1169-79.

[14] Tsukuma H, Mishima T, Oshima A. Prospective study of "early" gastric cancer. Int J Cancer 1983;31:421-6. 
[15] Kamangar F, Dawsey SM, Blaser MJ. Opposing risks of gastric cardia and noncardia gastric adenocarcinomas associated with Helicobacter pylori seropositivity. J Natl Cancer Inst 2006;98:1445-52.

[16] Helicobacter and Cancer Collaborative Group. Gastric cancer and Helicobacter pylori: a combined analysis of 12 case control studies nested within prospective cohorts. Gut 2001;49:347-53.

[17] Limburg P, Qiao Y, Mark S. Helicobacter pylori seropositivity and subsite-specific gastric cancer risks in Linxian, China. J Natl Cancer Inst 2001;93:226-33.

[18] Chow WH, Blaser MJ, Blot WJ. An inverse relation between cagA + strains of Helicobacter pylori infection and risk of esophageal and gastric cardia adenocarcinoma. Cancer Res 1998;58:588-90.

[19] Ye W, Held M, Lagergren JL. Helicobacter pylori infection and gastric atrophy: risk of adenocarcinoma and squamous-cell carcinoma of the esophagus and adenocarcinoma of the gastric cardia. J Natl Cancer Inst 2004;96:388-96.

[20] Correa P. Human gastric carcinogenesis: a multistep and multifactorial process-First American Cancer Society Award Lecture on Cancer Epidemiology and Prevention. Cancer Res 1992;52:6735-40.

[21] Hansson LR, Engstrand L, Nyren O, et al. Prevalence of Helicobacter pylori infection in subtypes of gastric cancer. Gastroenterology 1995;109:885-8.

[22] Ohata H, Kitauchi S, Yoshimura N. Progression of chronic atrophic gastritis associated with Helicobacter pylori infection increases risk of gastric cancer. Int J Cancer 2004;109:138-43.

[23] Japanese Gastric Cancer Association. Japanese classification of gastric carcinoma-2nd English edition. Gastric Cancer 1998;1:10-24.

[24] Tatsuta M, Iishi H, Okuda S, et al. The association of Helicobacter pylori with differentiated-type early gastric cancer. Cancer 1993;72: $1841-5$.

[25] Iseki K, Tatsuta M, Iishi H, et al. Helicobacter pylori infection in patients with early gastric cancer by the endoscopic phenol red test. Gut 1998;42:20-3.

[26] Sasazuki S, Inoue M, Iwasaki M. Effect of Helicobacter pylori infection combined with CagA and pepsinogen status on gastric cancer development among Japanese men and women: a nested case-control study. Cancer Epidemiol Biomarkers Prev 2006;15:1341-7.
[27] Karnes WE, Samloff IM, Siurala M. Positive serum antibody and negative tissue staining for Helicobacter pylori in subjects with atrophic body gastritis. Gastroenterology 1991;101:167-74.

[28] Wu AH, Crabtree JE, Bernstein L, et al. Roe of Helicobacter pylori $\mathrm{CagA}^{+}$straings risk of adenocarcinoma of stomach and esophagus. Int $\mathrm{J}$ Cancer 2003;103:815-21.

[29] Genta RM, Gurer IE, Graham DY. Adherence of Helicobacter pylori to areas of incomplete intestinal metaplasia in the gastric mucosa. Gastroenterology 1996;111:1206-1.

[30] Craanen ME, Blok P, Dekker W, et al. Helicobacter pylori and early gastric cancer. Gut 1994;35:1372-4.

[31] Linton PJ, Dorshkind K. Age-related changes in lymphocyte development and function. Nat Immunol 2004;5:133-9.

[32] Nakamura T, Yao T, Niho Y, et al. A clinicopathological study in young patients with gastric carcinoma. J Surg Oncol 1999;71:214-9.

[33] Lo SS, Kuo HS, Wu CW. Poorer prognosis in young patients with gastric cancer? Hepatogastroenterology 1999;46:2690-3.

[34] Yokota T, Takahashi N, Teshima S. Early gastric cancer in the young: clinicopathological study. Aust N Z J Surg 1999;69:443-6.

[35] Medina-Franco H, Heslin MJ, Cortes-Gonzalez R. Clinicopathological characteristics of gastric carcinoma in young and elderly patients: a comparative study. Ann Surg Oncol 2000;7:515-9.

[36] Matsui M, Kojima O, Kawakami S, et al. The prognosis of patients with gastric cancer possessing sex hormone receptors. Surg Today 1992;22:421-5.

[37] Furukawa H, Iwanaga T, Hiratsuka M. Gastric cancer in young adults: growth accelerating effect of pregnancy and delivery. J Surg Oncol 1994;55:3-6.

[38] Vohlonena I, Pukkala E, Malila N, et al. Risk of gastric cancer in Helicobacter pylori infection in a 15-year follow-up. Scan J Gastroenterol 2016;51:1159-64.

[39] Abadi ATB. Helicobacter pylori and Gastric Cancer. Front Med 2016;3:36.

[40] Lee Y-C, Chiang T, Liou JM, et al. Mass eradication of Helicobacter pylori to prevent gastric cancer: theoretical and practical considerations. Gut and Liver 2016;10:12-26. 